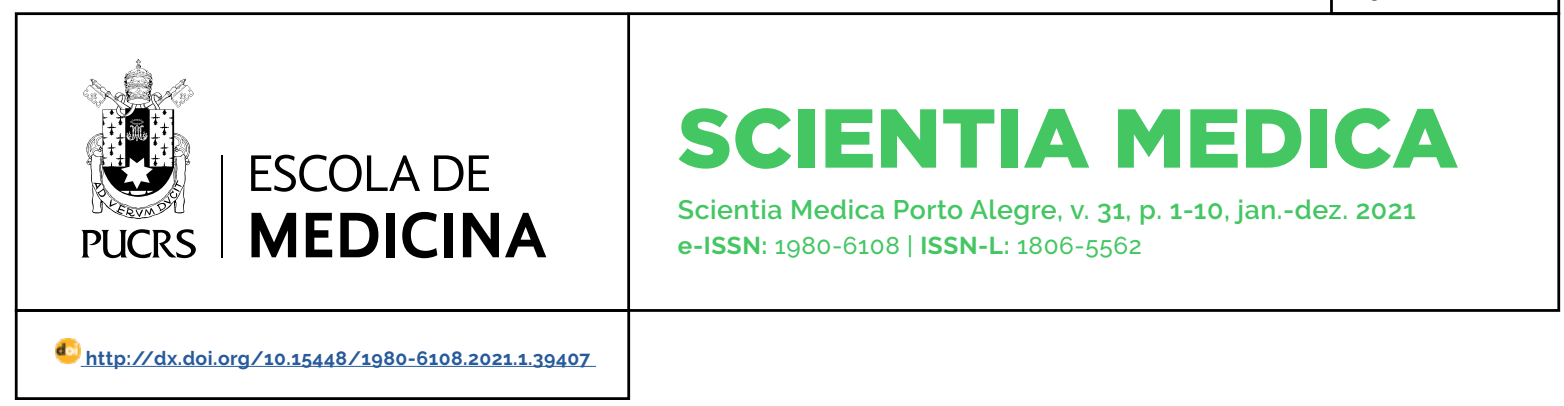

ARTIGO ORIGINAL

\title{
Aplicação da nota de transferência e do Paediatric Early Warning Score no serviço de emergência pediátrica do Hospital de Clínicas de Porto Alegre
}

\author{
Aplication of Transference Note and Paediatric Early Warning Score in the Pediatric \\ Emergency Department of Hospital de Clinicas de Porto Alegre
}

\section{Sofia Panato Ribeiro ${ }^{1}$}

orcid.org/0000-0002-0197-6385

sofispanato@gmail.com

Letícia Becker Vieira ${ }^{1}$ orcid.org/0000-0001-5850-7814

lebvieira@hcpa.edu.br

\section{Juliana Karine Rodrigues Strada ${ }^{1}$ orcid.org/0000-0002-9523-4584 julianakrstrada@gmail.com}

\section{Cássio Amaro Moreira} Freitas $^{2}$

orcid.org/0000-0003-3604-557X camfreitas@hcpa.edu.br

\section{Valmir Machado de Almeida ${ }^{2}$} orcid.org/0000-0003-2142-3005 vmalmeida@hcpa.edu.br

\section{Wiliam Wegner ${ }^{1}$}

orcid.org/0000-0002-0538-9655 wwegner@hcpa.edu.br

Recebido em: 22 out. 2020 Aprovado em: 4 ago. 2021. Publicado em: 29 out. 2021.

\section{Resumo}

Objetivo: avaliar a adesão dos enfermeiros ao preenchimento da Nota de Transferência com emissão do Paediatric Early Warning Score (PEWS) em pacientes pediátricos do Serviço de Emergência.

Método: estudo quantitativo, transversal e retrospectivo. O estudo foi realizado a partir da análise de 1.219 prontuários de pacientes de uma unidade de emergência pediátrica, no ano de 2018.

Resultados: na avaliação da nota de transferência e PEWS, houve adesão de, respectivamente, $86,8 \%$ e $75,2 \%$ ao longo do ano. Os motivos de hospitalização variam conforme época do ano. Os pacientes atendidos no Serviço de Emergência foram, majoritariamente, estabilizados no serviço e, somente os pacientes com PEWS alterado, foram encaminhados à unidade de terapia intensiva pediátrica.

Conclusões: são necessárias ações de educação com os profissionais, com a finalidade de melhoria dos indicadores relacionados à aplicação do PEWS e da nota de transferência para a garantia de uma assistência segura no que tange à continuidade do cuidado.

Palavras-chave: segurança do paciente, enfermagem pediátrica, pediatria,

\section{Abstract}

Aims: to evaluate the nurses' adherence to filling out the transfer note with the emission of the Paediatric Early Warning Score (PEWS) in pediatric patients in the Emergency Department.

Methods: quantitative, cross-sectional and retrospective study. The study has been conducted from the analysis of 1.219 medical records of patients in a Pediatric Emergency, in the year of 2018.

Results: in the evaluation of transference note and PEWS, the adherence was, respectively, $86,8 \%$ e $75,2 \%$, over the year. The reasons for hospitalization vary according to the time of the year. Most patients attended by the emergency department were stabilized at the emergency service and only the patients with altered PEWS were referred to the pediatric intensive care unit.

Conclusions: educational actions addressed to the professionals are necessary, with the objective to improve the indicators related the application of PEWS and the transfer note to guarantee safety assistance regarding the continuity of the care.

Keywords: patient safety, pediatric nursing, pediatrics. 


\section{Introdução}

O cuidado de enfermagem em pediatria é caracterizado por diversas situações que requerem mais atenção dos profissionais de saúde. A população pediátrica possui maior risco de eventos adversos, pois existem alguns aspectos especificos que influenciam na segurança do cuidado ao paciente, os quais podemos citar idade, fragilidade e vulnerabilidade em comparação à população adulta, pois (1). A segurança do paciente é compreendida como uma ação destinada a evitar ou prevenir eventos adversos decorrentes do processo de cuidado, sendo considerada uma dimensão da qualidade do atendimento. Deve ser valorizada como um direito do paciente e um compromisso ético do profissional, assim, o profissional é responsável pela efetividade da assistência e pelo cuidado centrado no paciente (2).

No que tange o paciente pediátrico, evidencia-se que um dos principais momentos que oferece risco para a ocorrência de incidentes e de eventos adversos da criança é na transferência do cuidado, pois qualquer informação, enviada ou interpretada incorretamente, pode acarretar diversos tipos de incidentes que comprometem a segurança do paciente (3). Entre as consequências das falhas na comunicação entre as equipes na transferência do cuidado, encontra-se a diminuição da qualidade dos cuidados e, como consequência, o dano à saúde e o aumento do tempo de hospitalização por erros dos profissionais (4).

No serviço em estudo, realiza-se em prontuário eletrônico a nota de transferência do paciente. Essa nota visa garantir a continuidade do cuidado e a segurança do paciente, contendo suas principais informações, como a utilização de oxigênio, tipo de dieta, sinais vitais, escore para avaliação da deterioração clínica do paciente, entre outros, sendo considerada uma estratégia para melhora da comunicação (5).

A deterioração clínica de um paciente é identificada a partir de sinais e de sintomas que demonstram sua instabilidade fisiológica. Escores que detectam precocemente alterações fisiológicas nos pacientes vêm sendo utilizados em larga escala nas práticas hospitalares. Esses, conhecidos como Early Warning System, são um conjunto de escalas numéricas, as quais quantificam uma associação de parâmetros, a partir da observação do profissional da saúde, apontando quando um parâmetro sai de seus valores de normalidade e as ações necessárias para a estabilização do paciente, como avisar o médico ou o enfermeiro imediatamente para estabelecimento de novas condutas, o que resulta na prevenção de complicações e sequelas, assim como na diminuição dos gastos públicos com internação hospitalar e tratamento $(6,7)$.

Em relação aos pacientes pediátricos, utilizam-se instrumentos denominados como Paediatric Early Warning Score (PEWS), escores que levam em consideração caracteristicas como frequência cardíaca, frequência respiratória, pressão arterial, enchimento capilar, esforço respiratório, saturação de oxigênio e utilização de oxigênio (7). Esses mecanismos têm como objetivo reduzir o risco de eventos adversos sérios, como parada cardiorrespiratória, entre outros. Entretanto, o escore considera como uma característica do ambiente pediátrico, que os parâmetros vitais das crianças variam de acordo com a sua faixa etária (6).

Após a identificação da piora clínica, é necessário um cuidado intensivo para com este paciente. Entretanto, em muitos ambientes hospitalares, não há o quantitativo de pessoal e materiais necessários para a realização dos cuidados adicionais, sendo este o principal motivo para a transferência de cuidado intra-hospitalar tanto em pacientes adultos, quanto pediátricos (4).

No ano de 2017, foi implementado no serviço de emergência pediátrica do hospital foco do estudo, a PEWS adaptada, a qual tem como objetivos estabelecer o processo de avaliação dos pacientes pediátricos hospitalizados, identificar futuras intercorrências e a necessidade de transferência de cuidado do paciente para a unidade de terapia intensiva pediátrica, além de sinalizar para uma atenção precoce, a fim de estabilizar o paciente.

Portanto, justifica-se o presente estudo pelas consequências advindas das falhas da comunicação, as quais podem causar danos significativos aos pacientes, pois quebram a continuidade do tratamento e a qualidade da assistência (3); e na importância da construção de uma cultura da segurança do paciente na atenção à saúde da 
criança hospitalizada por meio de produção de conhecimento para a elaboração de recomendações de estratégias para a promoção do cuidado seguro na enfermagem pediátrica.

Diante da problemática exposta sobre a comunicação efetiva como estratégia para a segurança do paciente tem-se a seguinte questão de pesquisa: "As notas de transferência e emissão do PEWS na unidade de emergência pediátrica em hospital de ensino estão sendo realizados de acordo com as diretrizes propostas pela instituição?"

Tem-se como objetivo geral do presente estudo avaliar a adesão dos enfermeiros ao preenchimento da nota de transferência com emissão do PEWS em pacientes pediátricos do setor emergência.

\section{Método}

Tratou-se de um estudo com abordagem quantitativa, do tipo transversal e retrospectivo. $O$ estudo foi realizado por intermédio da análise de prontuários de pacientes internados na unidade de emergência pediátrica do Hospital de Clínicas de Porto Alegre. O serviço é a porta de entrada do hospital, onde são atendidas crianças de 0 a 13 anos, 11 meses e 29 dias, sendo que seu atendimento ideal deve durar menos de 24 horas, pois deve-se atender situações caracterizadas como urgência ou emergência pediátricas. Realiza atendimento humanizado, pautado na inserção da família no ambiente de cuidado, assegurando o direito do acompanhante a permanecer junto à criança (5).

A emergência é composta pelos ambientes de acolhimento e de classificação de risco, dois consultórios, sala de procedimentos pediátricos, box de atendimento de urgências, box de admissão de pacientes e sala de observação pediátrica e três boxes de isolamentos. O serviço é formado por uma equipe multiprofissional, a qual inclui médicos, equipe de enfermagem, fisioterapeutas, nutricionistas, psicólogos, assistentes sociais, farmacêuticos e, ainda, recebe para estágio alunos da residência médica e alunos da graduação em enfermagem e medicina (5).

Em relação à equipe de enfermagem, é composta por um enfermeiro e três técnicos de enfermagem por turno (5). Apesar de aumentar significativamente o número de crianças à procura de atendimento nos meses com temperaturas mais baixas, não há alteração na composição da equipe conforme o número de pacientes. Quando há necessidade, há cobertura por profissionais de outras áreas do hospital para manutenção do atendimento.

Os pacientes podem dar entrada no serviço por demanda espontânea, encaminhados pelo serviço de atendimento móvel de urgência ou demais serviços pré-hospitalares e/ou encaminhados por demais serviços do hospital. Pautou-se na Política Nacional de Humanização, em que uma das diretrizes para o atendimento em urgências e emergências é o acolhimento com avaliação e classificação de risco. Para atender essa diretriz, o serviço de enfermagem em emergência adota o protocolo de Manchester (5).

A população de estudo foi composta dos pacientes pediátricos cujas transferências foram realizadas pelos enfermeiros ou estagiários de enfermagem que trabalharam no serviço, nos turnos da manhã, tarde e noite. A amostra foi composta por 1.219 prontuários de pacientes que foram transferidos da emergência pediátrica para outros setores do hospital, no período de 1 de janeiro a 31 dezembro de 2018.

Foram incluidos os pacientes de o meses a 13 anos, 11 meses e 29 dias de idade, de ambos os sexos. Foram excluidos, os pacientes que foram transferidos imediatamente após o primeiro atendimento na emergência para a unidade de tratamento intensivo pediátrico e neonatal visto que se sabe previamente que esses pacientes têm declínio clínico, logo, não é necessário mensurar a gravidade por meio do PEWS.

Assim, foi verificado o correto preenchimento das notas de transferência e a inclusão do PEWS. Em relação à nota de transferência, foi considerada completa quando todos os campos foram preenchidos e, incompleta, quando houve ausência de informação. Ela tem como objetivo informar, de maneira resumida, quais as condições do paciente à equipe que irá recebê-lo.

O PEWS é realizado a cada verificação de sinais vitais dos pacientes e implementado na nota de transferência. A escala varia de acordo com a faixa etária, abrangendo os grupos de crianças com idade <3 meses; de 3 a <12 meses; de 1 a $<5$ anos; de 5 a $<12$ anos e $>12$ anos (9). 
O PEWS adaptado para o Hospital de Clínicas de Porto Alegre, tem como sua pontuação mínima o pontos e, máxima, 26 pontos, sendo que pacientes com escore acima de 9 pontos já são considerados pacientes graves, com necessidade de avaliação médica urgente.
Como o serviço de emergência é uma porta de entrada hospitalar, é esperado que os pacientes internados no local tenham parâmetros fisiológicos alterados, sendo assim, os padrões de referência são mais altos em comparação aos demais serviços do hospital, como evidenciado na Tabela1.

TABELA 1 - Orientações para condutas conforme PEWS.

\begin{tabular}{ccccc}
\hline Escore & Sinais Vitais & Enfermeiro & Médico & Ação adicional \\
\hline o a 6 & $3 / 3 \mathrm{~h}$ & $3 / 3 \mathrm{~h}$ & $2 \times /$ dia & Não \\
7 a 8 & $2 / 2 \mathrm{~h}$ & $2 / 2 \mathrm{~h}$ & $3 \times /$ dia & Avisar enfermeira imediatamente \\
9 ou + & $1 / 1 \mathrm{~h}$ & $1 / 1 \mathrm{~h}$ & $1 / 1 \mathrm{~h}$ & Avisar médico imediatamente \\
\hline
\end{tabular}

PEWS, Paediatric Early Warning Score.

Fonte: Hospital de Clínicas de Porto Alegre (9).

Assim, indica-se a ação adicional conforme a pontuação do paciente, como a diminuição do tempo entre as verificações dos sinais vitais e o aviso ao enfermeiro e/ou ao médico imediatamente.

Os dados foram coletados do prontuário eletrônico dos pacientes pediátricos, cujas informações foram registradas na nota de transferência, por intermédio de acesso ao sistema informatizado. Subsequentemente, foram lançados em planilha do software Excel e, posteriormente, importados ao software SPSS para as análises pertinentes. Entre as variáveis estudadas, pôde-se identificar o turno de trabalho, mês e local de destino.

Foi realizada uma análise descritiva, sendo apresentada com uso de gráficos. Obteve-se aprovação no Comitê de Ética em Pesquisa da instituição sob número do parecer 3.403.556. A pesquisa fez parte do projeto matriz denominado "Escala de Avaliação de Deterioração Clínica na Melhoria da Segurança do Paciente Pediátrico".

\section{Resultados}

No ano de 2018, o serviço de emergência pediátrica do hospital realizou transferência intra-hospitalar de 1.219 pacientes. Os meses em que mais houve transferências foram junho, julho, agosto e dezembro. Na avaliação da nota de transferência, houve adesão dos enfermeiros de 86,84\% ao longo do ano. Entretanto, a adesão ficou acima de 90\% somente nos meses de dezembro, novembro, março e junho, respectivamente (Figura 1).

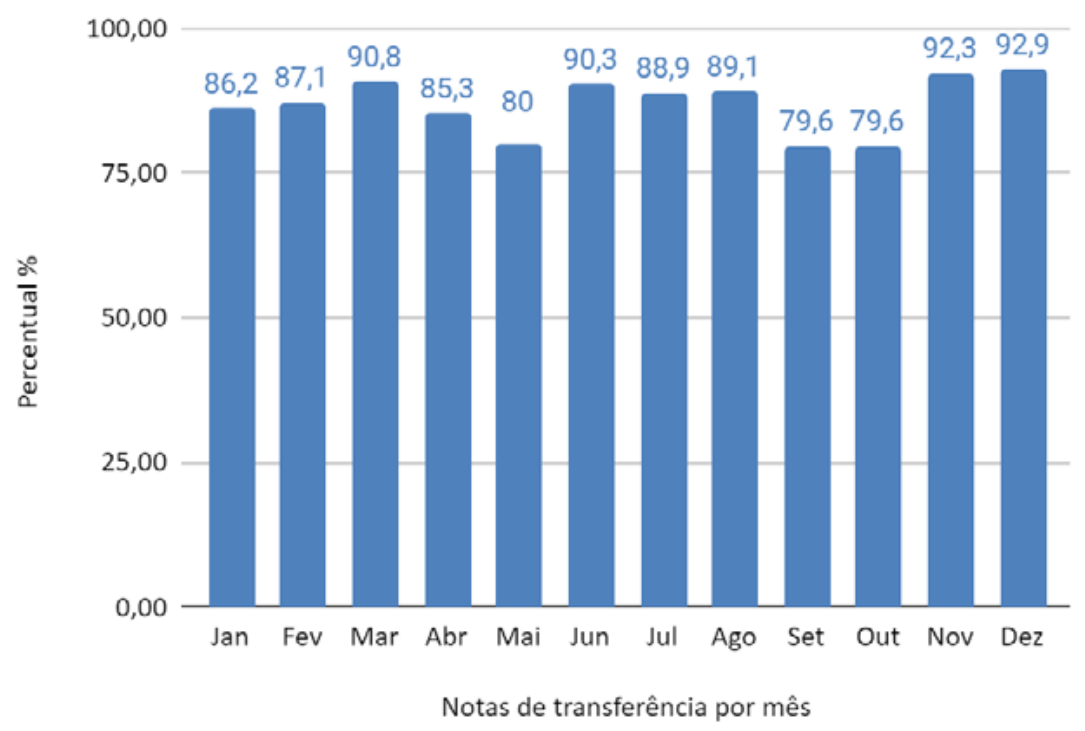

Figura 1 - Valores percentuais de notas de transferência realizadas ao mês no serviço de emergência, setor pediatria. 
Ao longo do ano em estudo, os turnos que realizaram maior adesão da nota de transferência foram, respectivamente, o turno da tarde $(n=449)$, noite ( $n=431)$ e manhã ( $n=179)$ (Figura 2).

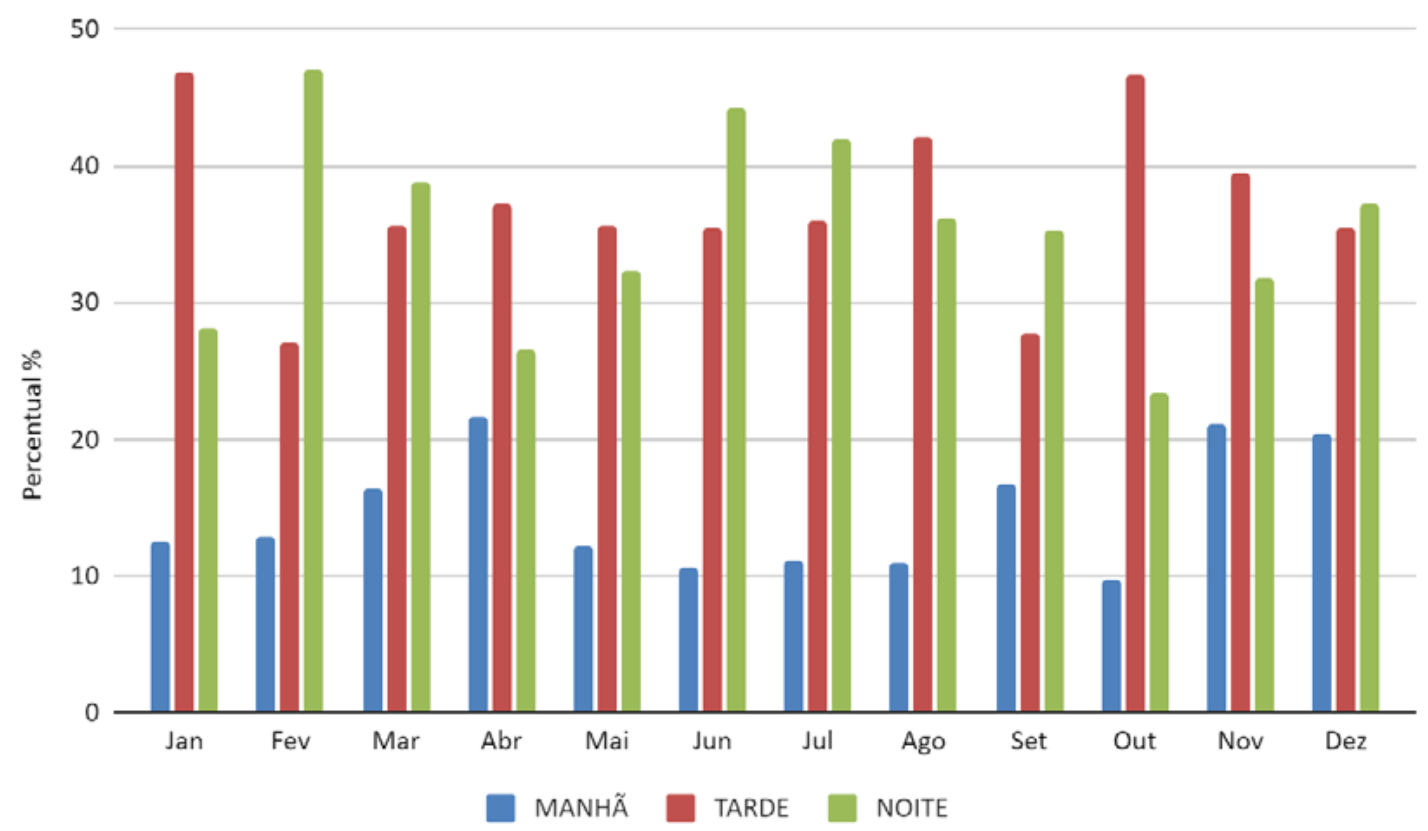

Figura 2 - Comparação da realização de notas de transferência pelos profissionais de cada turno.

Em relação ao PEWS, dos 1.219 pacientes transferidos, o escore foi mensurado em 916 $(75,24 \%)$ dos prontuários. Nos meses de março e abril, a mensuração do escore foi maior que $80 \%$, portanto, apesar dos valores serem significativos, há oportunidade de melhoria da adesão por parte da equipe (Figura 3).

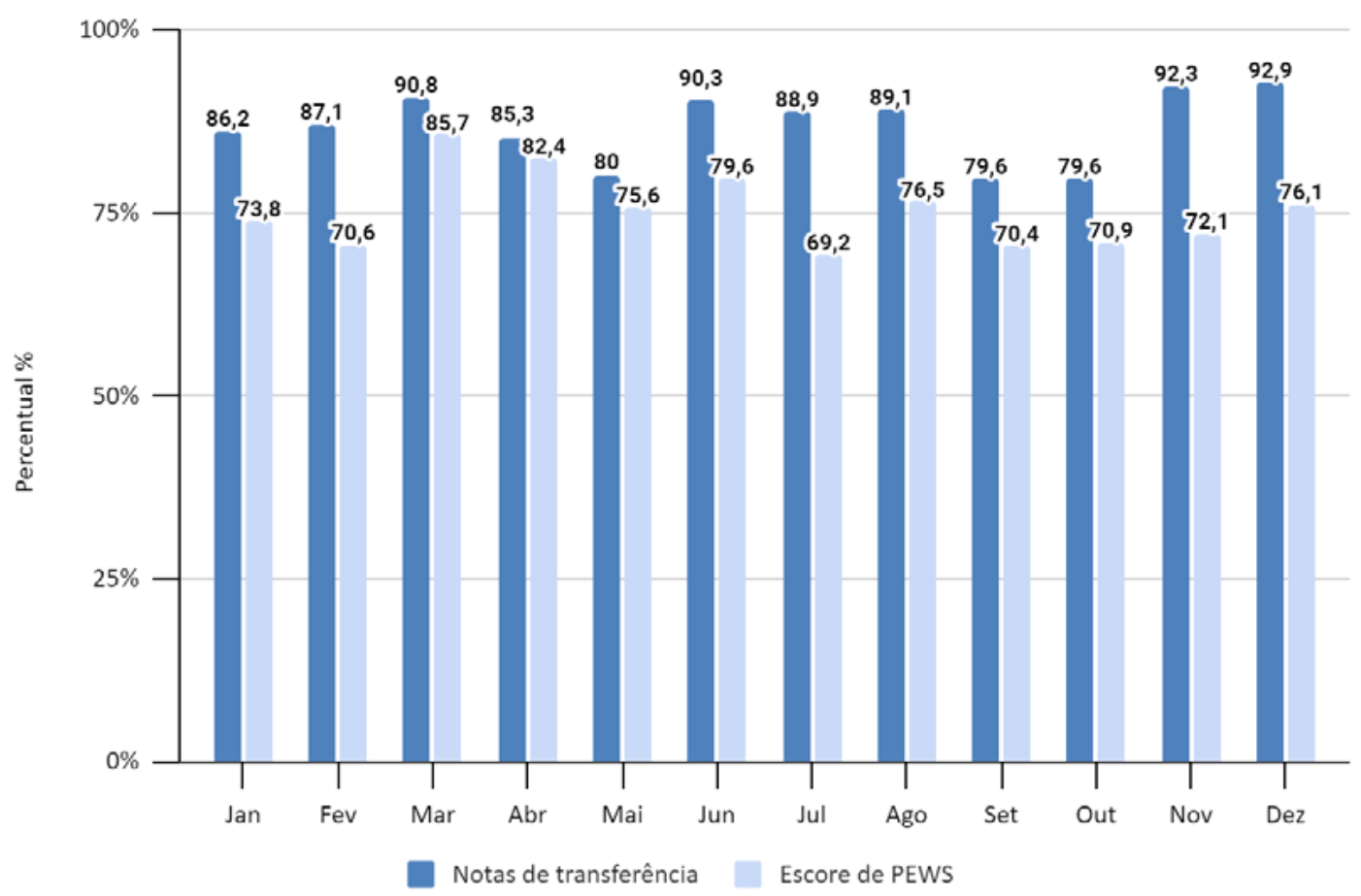

Figura 3 - Valores percentuais da realização de notas de transferência e do PEWS. 
Com os valores mensurados do escore, foi possivel verificar que 867 pacientes apresentaram pontuação não alterada, a qual varia de o a 6 e, 49 pacientes possuiam pontuação alterada, considerada quando $>7$.

Dos 1.219 pacientes que realizaram transferência no ano de 2018, observou-se que os destinos que mais receberam pacientes encaminhados da emergência pediátrica foram, respectivamente, 0 serviço de internação pediátrica, totalizando 844 transferências (69.3\%); oncologia pediátrica, com 129 transferências (10,6\%); unidade de tratamento intensivo pediátrico, com 109 transferências ( $9 \%$ ); bloco Cirúrgico, com 80 pacientes transferidos (6,6\%); unidade de tratamento intensivo neonatal, com 27 transferências (2,2\%); internação neonatal com 7 pacientes transferidos (0,6\%); centro cirúrgico ambulatorial, com 6 pacientes transferidos (0,5\%); outros, 15 transferências (1,2\%).

Em relação aos pacientes que foram encaminhados à internação pediátrica, 663 (78,5\%) possuiam PEWS no sistema e 651 (98\%) dos pacientes com a presença do escore em prontuário, apresentaram PEWS não alterado.

No que tange o destino dos pacientes com PEWS alterado (>7), verificou-se que 36 (73,4\%) foram transferidos à unidade de terapia intensiva pediátrica, 12 (24,4\%) para a unidade de internação pediátrica e $1(2,04 \%)$ para a oncologia pediátrica.

\section{Discussão}

Constatou-se que os meses em que mais ocorreram transferências intra-hospitalares de pacientes pediátricos do serviço de emergência para demais áreas assistenciais estão relacionados ao recorte sazonal do inverno, além do mês de dezembro. Isso decorre de características climáticas da região Sul do Brasil, pois os meses de junho, julho e agosto têm temperaturas mais baixas e, como consequência, um aumento dos agravos respiratórios; por outro lado, os meses de verão, como o mês de dezembro, têm incidência de casos de doenças virais. Entretanto, nos meses de janeiro e fevereiro ocorre migração para a região do litoral, diminuindo o número de atendimentos no hospital, localizado na capital do Estado.
Em um estudo realizado em um hospital de Portugal, onde investiga-se sobre o uso da sala de reanimação, demonstra-se que sua utilização também apresenta variações conforme o período do ano. O mês de julho apresenta menor quantidade de atendimentos, pois é um periodo de verão, no qual as crianças encontram-se em férias escolares. Por outro lado, os meses de janeiro e dezembro têm maior número de urgências relacionadas às doenças respiratórias, por ser período de inverno (10).

Na instituição, não há uma meta estabelecida para a realização do PEWS e da nota de transferência, entretanto, por se buscar maior qualidade no atendimento, tem-se como objetivo alcançar 100\% de adesão. Em relação à adesão dos enfermeiros à nota de transferência, é possivel verificar que, em sua maioria, os meses em que houve maior número de transferências, apresentaram taxa menor de adesão à nota de transferência (julho e agosto).

Estudo mostra que a falta de adesão dos profissionais de enfermagem a alguns processos de trabalho pode ser explicada por maior carga de trabalho dos profissionais, somado ao pequeno quantitativo de funcionários, principalmente em unidades de urgência e de emergência, onde há um grande fluxo de pacientes atendidos diariamente, o que disponibiliza menor tempo para realização das atividades de gerenciamento do cuidado (11), como a realização da nota de transferência. Assim, é possivel relacionar a falta de adesão da equipe ao pequeno número de profissionais na unidade, pois não há alteração no número de funcionários, apesar do aumento significativo do número de pacientes durante o período identificado.

Segundo achados do estudo, o turno da tarde obteve maior adesão à nota de transferência em relação aos turnos da noite e da manhã. Isso ocorre como consequência de algumas características de cada turno e da especificidade do processo de trabalho do hospital em tela, em que a decisão clínica relativa às transferências ocorre em maior volume no periodo da tarde.

Em comparação aos demais estudos, evidencia-se que a maioria dos atendimentos em um serviço de emergência pediátrica ocorreu no 
período referente ao turno da tarde, seguido do período referente ao turno da noite (10) e constata-se que em um hospital com grande vulnerabilidade social, a maior taxa de atendimentos ocorre no turno da tarde, pois há maior disponibilidade de horários dos pais para levarem seus filhos ao serviço de saúde (12). Assim, podemos associar essa caracteristica à maior necessidade de transferências neste turno, em especial.

Visualiza-se que a realização da nota de transferência está mais consolidada na equipe de saúde, quando comparada com a realização do PEWS. Podemos relacionar essa afirmação com a recente implementação do escore na instituição, pois a nota de transferência é realizada no sistema informatizado desde o ano de 2015.

Os dados da pesquisa foram coletados após o primeiro ano de implementação do PEWS na instituição de saúde. Assim, além de ser um processo de trabalho recente, o escore foi implantado de forma manual e deveria ser anexado à nota de transferência no sistema informatizado, sendo um dos motivos para falta de adesão dos enfermeiros da equipe. Assim, no mês de novembro do ano de 2018, os sinais vitais utilizados para o cálculo do escore também passaram a ser informatizados, entretanto, seu cálculo continuava sendo manual. Somente no mês de janeiro de 2019, o cálculo do escore também passou a ser informatizado, necessitando de nova análise para constatar se houve maior adesão após a informatização.

Estudo afirma que há inúmeros benefícios em informatizar a sistematização da assistência de enfermagem, por exemplo, garante maior segurança aos registros do paciente, além de garantir tempo reduzido para documentar as informações, o que proporciona uma maior adesão às tarefas de gerenciamento do cuidado (13). Assim, observa-se oportunidade de melhorias em relação ao processo, com a progressão da informatização dos dados em prontuário, o que tende a uma maior adesão ao PEWS juntamente à nota de transferência.

Além disso, a importância da realização do PEWS deve-se a uma característica institucional atribuída a esse escore, pois, no hospital de estudo, é verificada a pontuação a cada aferição de sinais vitais, sendo realizada, no mínimo, duas vezes ao turno, além de ser utilizada no momento de transferência do cuidado.

Assim, torna-se uma ferramenta de segurança para o paciente, a qual sinaliza para a equipe que está recebendo, o estado de saúde, de maneira objetiva. A informatização também contribui com o acesso à informação, pois pode-se verificar o prontuário do paciente de qualquer unidade, tornando-se um instrumento facilitador para troca de informações e, como consequência, contribui para a melhor comunicação entre a equipe de saúde dos diferentes setores da instituição, proporcionando a rápida informação no caso de transferência interna do paciente, favorecendo o planejamento da equipe de enfermagem (13).

O hospital de estudo é conhecido como referência para o tratamento de doenças clínicas, genéticas e oncológicas, pois não faz atendimento de emergência traumática. A partir das informações coletadas, evidencia-se que os pacientes transferidos apresentam doenças clínicas, sendo, principalmente, doenças do aparelho respiratório e demais doenças, os quais são encaminhados à unidade de internação pediátrica, e doenças oncológicas, os quais são encaminhados à oncologia pediátrica. Essas duas unidades aparecem no gráfico como os dois destinos que mais recebem pacientes transferidos do serviço de emergência.

Conforme estudo relacionado aos motivos que levam à internação de pacientes pediátricos, as principais patologias identificadas nos pacientes atendidos em unidades de emergência pediátrica foram as doenças respiratórias, doenças gastrointestinais e as viroses virais. O maior número de internações ocorreu na unidade de internação pediátrica, seguido da unidade de oncologia pediátrica, da unidade de terapia intensiva pediátrica, da unidade de internação neonatal e da unidade de terapia intensiva neonatal (14).

Além disso, as unidades de terapia intensiva pediátrica e neonatal não apresentam grandes percentuais de recebimento de pacientes do setor. Entretanto, ao analisar os pacientes com PEWS alterado, foi possivel constatar que 
a maior prevalência é de encaminhamentos para a unidade de terapia intensiva pediátrica (73,4\%). Assim, foi possivel verificar que os pacientes são estabilizados na emergência, sempre que possivel e, somente os pacientes mais instáveis e/ ou com prognósticos ruins são encaminhados à unidade de terapia intensiva.

Também foi possivel observar que, em relação aos pacientes que apresentaram PEWS alterado, 24,4\% foram transferidos para a unidade de internação pediátrica. Essa informação pode evidenciar a necessidade de liberação dos leitos de urgência, assim como pode estar relacionada a outras situações, como a presença de doenças prévias que ocasionam alterações nos sinais vitais, o que não implica na instabilidade hemodinâmica do paciente. Entretanto, vale destacar que o PEWS recomenda que a transferência intra-hospitalar seja realizada após a estabilização do paciente.

Após esta análise, conseguimos observar que o PEWS garante um cuidado qualificado ao paciente, pois é possivel ver a relação entre encaminhamentos de pacientes com pontuação alterada para setores fechados e, entre os pacientes com pontuação não alterada, encaminhados à unidade de internação.

Além disso, também sinaliza maiores cuidados no transporte do paciente crítico durante sua transferência, possibilitando uma organização em relação à formação da equipe e aos materiais necessários no momento do transporte, proporcionando maior segurança para o profissional e o paciente.

Portanto, podemos evidenciar que instrumentos de alerta precoce auxiliam o trabalho da equipe de saúde no geral, mas, principalmente, no trabalho da enfermagem, contribuindo para que ofereçam um atendimento de qualidade, mais seguro, e auxiliem na alocação adequada da equipe de saúde, conforme a real necessidade de cuidados do paciente (15).

\section{Conclusão}

A nota de transferência e o PEWS são instrumentos que proporcionam mais segurança aos profissionais da saúde, principalmente à equipe de enfermagem, pois permitem a padronização da comunicação e a identificação de deterioração clínica do paciente pediátrico. Essas caracteristicas contribuem para melhora na continuidade do cuidado ao paciente desde sua entrada no serviço de emergência no atendimento imediato até o momento da transferência de cuidado. Além disso, auxiliam no gerenciamento em relação aos recursos humanos e materiais da unidade, proporcionando um melhor ambiente de trabalho aos profissionais e, consequentemente, diminuindo os riscos à saúde do paciente.

Foi possivel constatar que os profissionais não estão atingindo plena adesão aos instrumentos avaliados, portanto, há possibilidade de melhora dos indicadores da unidade, buscando atingir o seu potencial máximo. Entre os motivos que afetam a adesão, podemos citar a não informatização do PEWS e a grande carga de trabalho durante os meses em que ocorre maior lotação do serviço de emergência. Por outro lado, podemos concluir que a característica institucional atribuída ao PEWS em utilizá-lo conforme a realização dos sinais vitais facilita a adesão e, somando à informatização ocorrida no mês de dezembro, cria expectativas de uma maior adesão futura.

Assim, recomenda-se que toda a equipe faça o uso dos instrumentos estudados, para que ocorra uma linguagem comum entre todos os profissionais que prestam cuidado aos pacientes. Pensando-se do ponto de vista da gestão, com a finalidade de aumentar a adesão aos respectivos instrumentos, é necessário um planejamento de ações em educação continuada, além de adequá-los à realidade do serviço. Por exemplo, com o uso de ferramentas adaptadas à unidade. pensando em maneiras de otimizar o tempo do enfermeiro, em especial, no contexto de emergência, por sua grande demanda de trabalho. Além disso, são necessários mais estudos que avaliem a sensibilidade do PEWS para avaliar a deterioração clínica do paciente pediátrico, principalmente, no âmbito de emergência hospitalar.

Como limitadores do estudo, pode-se citar a situação abordada, a qual foi observada somente ao longo do primeiro ano de implantação do instrumento no hospital, além do processo manual 
na maior parte do periodo. Assim, acredita-se que em nova análise, a partir do segundo ano de implantação, haverá melhora dos indicadores institucionais. Além disso, é importante ressaltar que a aplicabilidade em outros serviços é limitada, pois sua aplicabilidade varia conforme o perfil dos pacientes atendidos, assim, seriam necessários mais estudos em diferentes serviços e instituições.

Portanto, concluímos que o PEWS e a nota de transferência são ferramentas importantes para a enfermagem, pois apresentam garantia de uma assistência segura. Assim, a maior adesão aos indicadores, por parte da equipe de enfermagem, reflete diretamente em menor risco de intercorrências na transferência intra-hospitalar, contribuindo para uma maior segurança do paciente. Além disso, propicia mais embasamento ao enfermeiro, a partir da apresentação de dados objetivos, tendo como consequência uma comunicação mais efetiva ao longo de todo o atendimento ao paciente e, principalmente, na transferência do cuidado.

\section{Notas}

Parte desse estudo foi publicado nos Anais do XII Congresso Gaúcho de Atualização em Pediatria, organizado pela Sociedade de Pediatria do Rio Grande do Sul e realizado nos dias 18 e 19 de setembro de 2020 .

\section{Apoio financeiro}

Este estudo não recebeu apoio financeiro de fontes externas.

\section{Declaração de conflito de interesses}

Os autores declaram não haver conflitos de interesses relevantes ao conteúdo deste estudo.

\section{Contribuições dos autores}

Todos os autores fizeram contribuições substanciais para concepção, ou delineamento, ou aquisição, ou análise ou interpretação de dados; e redação do trabalho ou revisão crítica; e aprovação final da versão para publicação.

\section{Disponibilidade dos dados e responsabilidade pelos resultados}

Todos os autores declaram ter tido total acesso aos dados obtidos e assumem completa responsabilidade pela integridade destes resultados.

\section{Referências}

1. Silva MF, Anders JC, Rocha PK, Souza Al, Burciaga VB. Communication in nursing shift handover: pediatric patient safety. Texto Contexto Enferm. 2016;25(3):e3600015. https://doi.org/10.1590/0104$\underline{07072016003600015}$

2. Bezerra AL. Editorial: A segurança do paciente e a enfermagem. Rev Nursing [Internet]. 2018 [citado 2021 Out 15];21(239):2091. Disponivel em: http://www.revistanursing. com.br/revistas/239-Abril2018/editorial.pdf

3. Bica TF, Wegner W, Gerhardt LM, Predebon CM, Pedro EN. Breigeiron MK. Características dos incidentes de segurança do paciente notificados em uma unidade de terapia intensiva pediátrica. Rev enferm UFPE on line [Internet]. 2017 [citado 2021 Out 15];11(Supl. 10):4206-16. https://doi.org/10.5205/1981-8963V11|10A231184P4206-4216-2017

4. Pires AF, Santos BN, Santos PN, Brasil VR, Luna AA. Transporte seguro de pacientes criticos. Revista Rede de Cuidados em Saúde [Internet]. 2015 [citado 2021 Out 15]:9(2):1-4. Disponivel em: http://publicacoes.unigranrio. edu.br/index.php/rcs/article/view/2531/1313

5. Hospital de Clínicas de Porto Alegre (BR). Plano assistencial do serviço de emergência pediátrica. 2016.

6. Roland D, McCaffery K, Davies F. Scoring systems in paediatric emergency care: panacea or paper exercise? J Paediatr Child Health. 2016:52(2):181-86. https://doi. org/10.1111/jpc.13123

7. Miranda JO, Camargo CL, Sobrinho CL, Portela DS, Monaghan A, Freitas K, et al. Tradução e adaptação de um escore pediátrico de alerta precoce. Rev Bras Enferm. 2016;6(5):888-96. http://dx.doi.org/10.1590/0034-7167$\underline{2015-0096}$

8. Miranda JO, Camargo CL, Sobrinho CL, Portela DS, Monaghan A. Acurácia de um escore pediátrico de alerta precoce no reconhecimento de deterioração clínica. Rev Latino-Am Enfermagem. 2017;25:e2912. http://dx.doi.org/10.1590/1518-8345.1733.2912

9. Hospital de Clínicas de Porto Alegre. Plano de Avaliação de pacientes pediátricos hospitalizados utilizando o escore Bedside Paediatric Early Warning Score (PEWS); 2018.

10. Macedo MA, Escudeiro M, Rodrigues L, Martins P, Sobral A. A sala de reanimação pediátrica: que realidade? Uma análise ao periodo 2015-2017. Rev Clin Hosp Prof Dr Fernando Fonseca [Internet]. 2017 [citado 2021 Out 15]:5(1/2):19-23. Disponivel em: https://revistaclinica. hff.min-saude.pt/index.php/rhff/article/view/235/130 
11. Costa ES, Silva MJ, Kuroba LS, Da Silva AM, Costa GS, Vieira PS. Processo de enfermagem em unidades de atendimento de urgência e emergência: uma revisão integrativa. Revista Uningá [Internet]. 2017 [citado 2021 Out 15];53(1):90-5. Disponivel em: http://revista.uninga. br/index.php/uninga/article/view/1407/1022

12. García JL, Orgeira JM, Peromingo JA. Demanda asistencial de urgencias pediátricas atendidas en un hospital comarcal. Atención Primaria. 2008;40(6):297301. https://doi.org/10.1157/13123682

13. Ribeiro JC, Ruoff AB, Baptista CL. Informatização da sistematização da assistência de enfermagem: avanços na gestão do cuidado. J Health Inform [Internet]. 2014 [citado 2021 Out 15];6(3):75-80. Disponivel em: http:// www.jhi-sbis.saude.ws/ojs-jhi/index.php/jhi-sbis/ article/view/296/199

14. Amthauer C, Cunha ML. Sistema de triagem de Manchester: principais fluxogramas, discriminadores e desfechos dos atendimentos de uma emergência pediátrica. Rev. Latino-Am. Enfermagem. 2016;24:e2779. http://dx.doi.org/10.1590/1518-8345.1078.2779

15. Pereira R, Mansur DG, Ionemoto HF. Implantação de escore de alerta de gravidade precoce em hospital infantil privado: relato de experiência. Rev. Soc. Bras. Enferm. Ped [Internet]. 2016 [citado 2021 Out 15];16(2):814. Disponivel em: https://sobep.org.br/revista/images/ stories/pdf-revista/vol16-n2/vol_16_n_2-relato_de_ experiencia_2.pdf

\section{Sofia Panato Ribeiro}

Enfermeira pela Universidade Federal do Rio Grande do Sul (UFRGS), em Porto Alegre, RS, Brasil; enfermeira residente na Residência Integrada Multiprofissional em Saúde (RIMS), programa Saúde da Criança, do Hospital de Clinicas de Porto Alegre (HCPA), RS, Brasil.

\section{Letícia Becker Vieira}

Doutora em Enfermagem pela Escola de Enfermagem Anna Nery da Universidade Federal do Rio de Janeiro (EEAN/UFRJ), no Rio de Janeiro, RJ, Brasil; professora Adjunta da Escola de Enfermagem da Universidade Federal do Rio Grande do Sul (EE-UFRGS), em Porto Alegre, RS, Brasil.

\section{Juliana Karine Rodrigues Strada}

Mestre em Enfermagem pela Universidade Federal do Rio Grande do Sul (UFRGS), em Porto Alegre, RS, Brasil.

\section{Cássio Amaro Moreira Freitas}

MBA em Gestão Hospitalar, escola São Camilo, em Porto Alegre, RS, Brasil; enfermeiro assistencial da Emergência Pediátrica do Hospital de Clínicas de Porto Alegre (HCPA), em Porto Alegre, RS, Brasil.

\section{Valmir Machado de Almeida}

Mestre em Educação pela UNILASALLE, em Canoas, RS; professor na Faculdade de Tecnologia do Instituto Pró Universidade Canoense (FATIPUC), em Canoas, RS; enfermeiro assistencial na Emergência Pediátrica do Hospital de Clínicas de Porto Alegre, em Porto Alegre, RS, Brasil.

\section{Wiliam Wegner}

Doutor em Enfermagem pelo Programa de PósGraduação em Enfermagem da Universidade Federal do Rio Grande do Sul (UFRGS), em Porto Alegre, RS, Brasil; professor adjunto da Escola de Enfermagem da UFRGS, em Porto Alegre, RS, Brasil.

\section{Endereço para correspondência}

Sofia Panato Ribeiro

Universidade Federal do Rio Grande do Sul

Rua Ramiro Barcelos, 2350

Porto Alegre, RS, Brasil

Os textos deste artigo foram revisados pela Poá Comunicação e submetidos para validação do(s) autor(es) antes da publicação. 